\title{
Diagnostic dilemma: an unusual case of primary intracranial, intraparenchymal epithelioid hemangioma
}

\begin{abstract}
Background and importance: Epithelioid hemangiomas, also known as angiolymphoid hyperplasia with eosinophilia and histiocytoid hemangioma, are benign vascular tumors that typically occur in the skin and subcutis. A primary intracranial presentation of this entity is unique. Radiologically, it can be confused with other more common vascular pathologies, such as metastasis, arteriovenous malformations $(\mathrm{AVM})$, or thrombosed aneurysms. Appreciation of this clinically benign vascular entity is important, as surgical resection is curative.
\end{abstract}

Clinical presentation: We present the case of a 57-year-old patient with a history of migraine headaches, syncope, seizures and thyroid cancer. The patient was recently involved in a car accident, and subsequently developed headaches that originated in the posterior cervical region and radiated to the occiput. CT scan of the head revealed a right temporal lobe mass, with surrounding edema. MRI showed a rounded mass with heterogeneous signal intensity, and magnetic susceptibility artifact on gradient echo T2-weighted sequences. Furthermore, the lesion was adjacent to the right middle cerebral artery bifurcation and a right M2 branch was noted to course through the lesion. The differential diagnosis included metastasis, thrombosed aneurysm, and arteriovenous malformation (AVM). The patient underwent a gross total surgical resection. The final pathological diagnosis was epithelioid hemangioma.

Conclusion: To our knowledge, this is the first reported case of a primary intracranial, intraparenchymal epithelioid hemangioma. It should be included in a differential list of intracranial vascular pathologies.

Keywords: ct angiography (cta), epithelioid hemangioma, intracranial, intraparenchymal, $\mathrm{mr}$ angiography (mra), magnetic resonance imaging (mri), vascular
Volume 8 Issue 5 - 2018

Srikant S Chakravarthi,' Srivathsa Veeraraghavan, ${ }^{2}$ Melanie Fukui,' Jonathan Jennings, ${ }^{2}$ Richard A Rovin,' Amin B Kassam' 'Department of Neurosurgery,Aurora Neuroscience Innovation Institute, USA

${ }^{2}$ Department of Radiology,Aurora St. Luke's Medical Center, USA

Correspondence: Amin B Kassam, Aurora Neuroscience Innovation Institute, 280 I West Kinnickinnic River, Parkway Milwaukee, USA,WI 53215, Email kassamab@gmail.com

Received: January 02, 2018 | Published: October 05, 2018

\section{Introduction}

Epithelioid hemangiomas (EH) are benign vascular tumors that more commonly occur in the skin, subcutis, and bone. ${ }^{1}$ Often confused with epithelioid hemangioendothelioma (EHE), EH is actually a lowgrade lesion along a spectrum of vascular lesions that includes the intermediate grade epithelioid hemangioendothelioma and high-grade epithelioid angiosarcoma. Although several cases of intracranial EHEs have been reported, we are not aware of a report of an isolated $\mathrm{EH}$ within the brain in the absence of skin or bone lesions. We present a case of this extremely unusual and rare entity.

\section{Case report}

This is a 57-year-old, with a history of migraine headaches, syncope, seizures and thyroid carcinoma, who presented to the Neurosurgery clinic following a car accident. The patient developed pain in the posterior cervical region that radiated to the occiput. These headaches were different in character from the typical migraine headaches.

Non-contrast CT scan of the head upon presentation revealed a 10X10X12mm right temporal lobe mass with associated edema. Mass effect was also noted upon the adjacent sulci. The mass effect and edema raised suspicion of a metastatic process. MRI revealed a rounded, enhancing lesion of heterogeneous signal intensity with central areas of bright and low signal on a T1 basis. There was intense magnetic susceptibility artifact on gradient echo T2-weighted sequences, suggesting hemosiderin deposition. There was no evidence of flow-related enhancement or prominent flow voids to suggest an arteriovenous malformation (AVM). Review of MRI performed five months earlier revealed that the lesion was only $5 \mathrm{~mm}$ in diameter without surrounding edema (Figure 1).
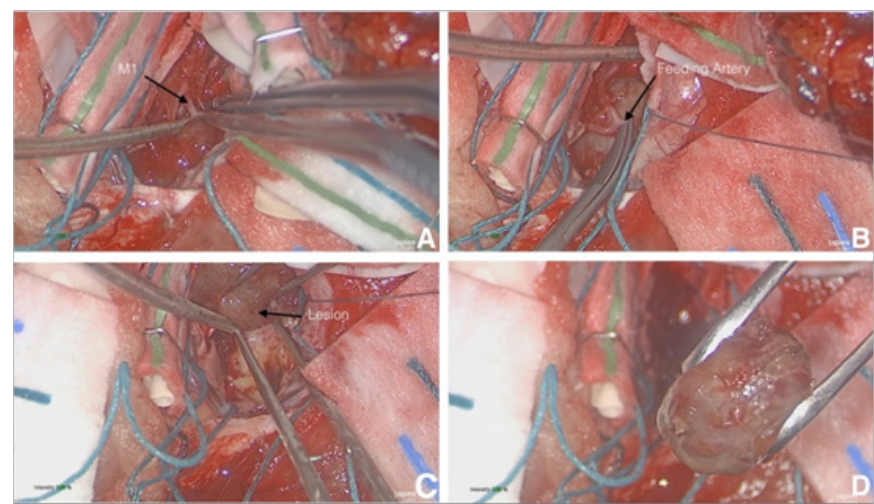

Figure I (A) Baseline axial T2 FLAIR MR image demonstrates a $6 \mathrm{~mm}$ lesion adjacent to the mesial temporal lobe in the Sylvian cistern, immediately adjacent to the MCA bifurcation, with no significant associated edema. (B) Follow up at 5 months demonstrates development of vasogenic extending into temporal white matter and temporal stem. (C) Follow up at 6 months at a slightly cranial level demonstrates worsening vasogenic edema now extending as far medial as the internal capsule. 
MR angiography (MRA) and CT angiography (CTA) was subsequently performed. MRA demonstrated mild T1 shortening without definite flow-related enhancement. CTA revealed a $1 \mathrm{~cm}$ intensely enhancing focus in the right temporal lobe, at the trifurcation of the right middle cerebral artery. The lesion demonstrated comparable enhancement to the intracranial vasculature. A right M2 branch appeared to course through this lesion, with a faint peripheral blush. There was no evidence of intracranial hemorrhage to indicate rupture (Figure 2). Catheter angiography did not demonstrate a vascular lesion.

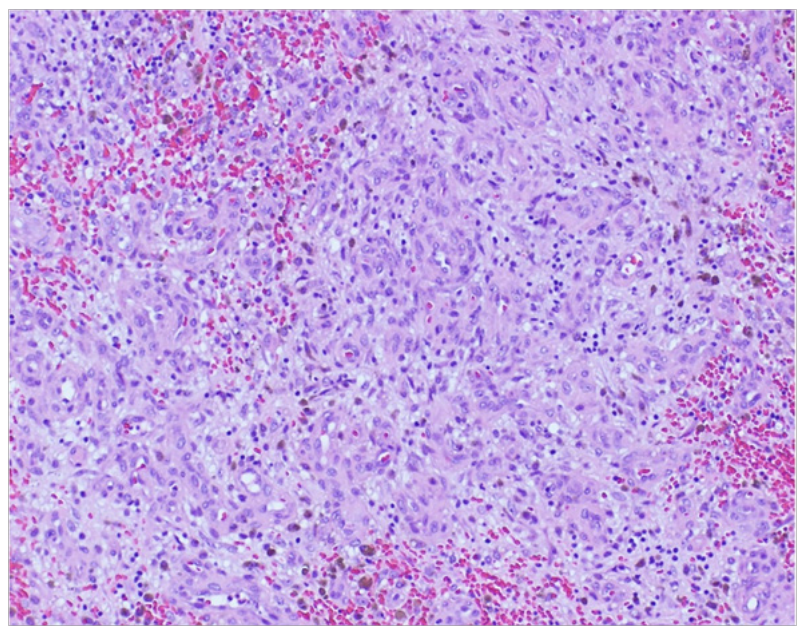

Figure 2 Baseline 3D time-of-flight MRA (A) and subvolume MIP MRA (B) images demonstrate mild TI shortening without definite flow-related enhancement (white arrows). Delayed axial image from a CT angiogram at 5 months (C) demonstrates interval increase in size to $6 \mathrm{~mm}$ with delayed enhancement (black arrow). 3D volumetric reconstructions from a catheter selective right ICA angiogram at 5 months (D) demonstrate no evidence of arterial flow to the lesion.
Because the lesion increased in size and was now associated with surrounding vasogenic edema (Figure 3), and there was no evidence of vascular pathology, the primary diagnostic consideration was metastasis. A decision was then made to surgically resect the lesion.

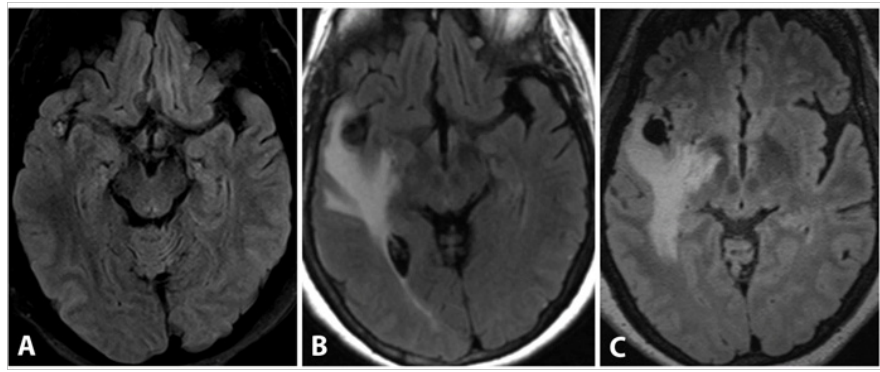

Figure 3 3D T2 FLAIR at 6months from baseline (A) demonstrate worsening vasogenic edema. 3D FLAIR at I month post resection (B) demonstrate complete resection of the lesion with resolution of vasogenic edema and minimal residual post-operative blood product.

\section{Operative technique}

The patient was brought to the operating room for the purposes of diagnosis and cytoreduction. A trajectory centric approach using real time 3D DTI rendering was created (BrightMatter Plan $\mathbb{C}$, Synaptive Medical Corporation, Toronto, Canada). A transsylvian corridor was ultimately chosen in order to optimize safe resection of the lesion. A robotic optical microscope (Synaptive Medical Corporation, Toronto, Canada) was used for visualization. Using standard microdissection technique and image guidance, the Sylvian fissure was opened proximal to distal. The M1 segment was then isolated. After the circumferential dissection of the mass, an arterial afferent was noted. The pedicle artery was isolated and it ended in the mass itself without an identifiable efferent branch. This pedicle was coagulated and divided. The lesion was then delivered en bloc (Figure 4).

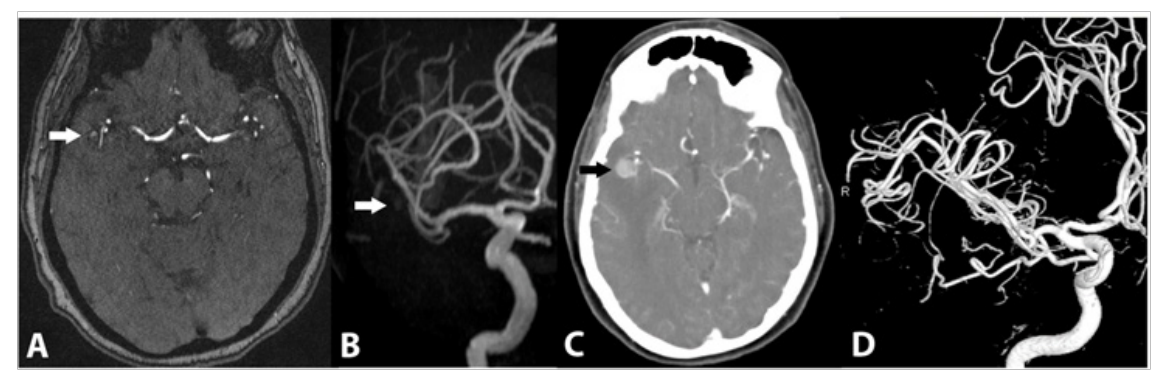

Figure 4 Surgical resection using standard microdissection techniques and image guidance. (A) The MI segment was isolated. (B) After the circumferential dissection of the mass, an arterial afferent was discovered. The feeding pedicle artery was then isolated, terminating in the mass itself. (C) After the artery was divided, the mass was removed en bloc (D).

\section{Pathological findings}

Histology showed a relatively well-circumscribed vascular proliferation consisting of well-formed capillary sized blood vessels lined by epithelioid cells and embedded within a fibromyxoid stroma containing mixed acute and chronic inflammatory cells. Focal areas imparted a solid-sheet like appearance due to compression of vessels. Scattered, larger caliber, hyalinized blood vessels and extensive hemosiderin deposition were present (Figure 5). Immunohistochemical staining revealed CD31 positivity, which highlighted the presence of well-formed vessels and the lining of endothelial cells. The lesional cells were negative for S-100, GFAP, inhibin, and CAM 5.2. The histomorphology and immunohistochemical profile yielded a final diagnosis of epithelioid hemangioma.

\section{Patient outcome}

The patient recovered from surgery without major complications.
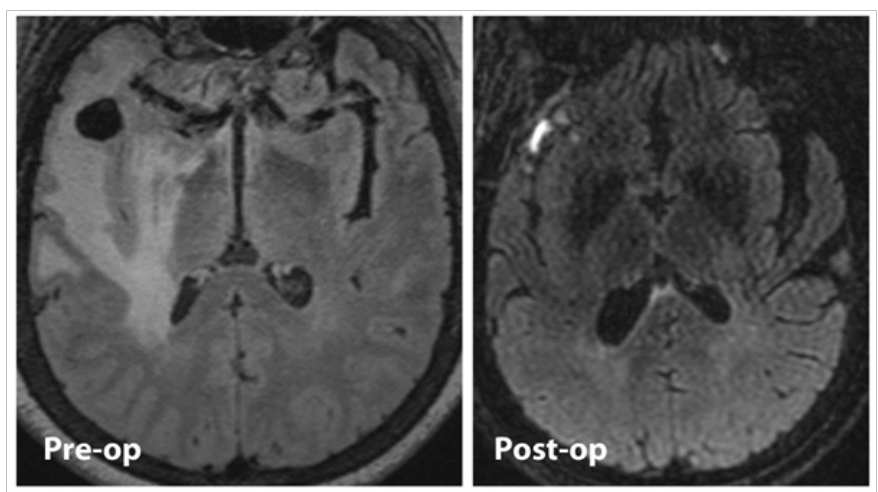

Figure 5 Pathology slide ( $\mathrm{H}$ and $\mathrm{E}$ staining) of tissue specimen showing hyalinized blood vessels in a solid sheet-like appearance, as well extensive hemosiderin deposition. 


\section{Discussion}

Primary epithelioid hemangioma of the brain is an extremely rare entity. Osteraas et al., recently described a case of multiple intracranial EHs with associated pigmented nodular skin lesions. However, the manifestation of an intracranial lesion without associated systemic manifestations, such as skin or bone lesions, has not yet been reported to our knowledge.

The differentiation of epithelioid hemangiomas from other vascular tumors (especially hemangioendotheliomas) is in their clinical behavior, histologic features, and the absence of gene rearrangements by $\mathrm{FISH}^{2}$ EHs have often been mistaken with EHEs in terms of histopathological features. Grossly, EHs appear well-circumscribed, lobular, soft, and hemorrhagic in consistency. Microscopically, they have small caliber capillaries with a granular, eosinophilic vacuolated cytoplasm, and large, oval, grooved, lobulated nuclei. ${ }^{3}$ The features of endothelial cells and angiogenesis are shared with epithelioid hemangioendothelioma. EH, however, is distinguished by the presence of mature, well-formed vessels with open lumina with surrounding epithelioid endothelial cells. In contrast, EHEs demonstrate less welldefined lumina, within a myxoid-hyaline stroma. ${ }^{4}$

With respect to imaging features, EHs are typically low in signal intensity on T1-weighted images and of intermediate signal intensity on T2-weighted images. ${ }^{5}$ Bone lesions often appear lytic, septated, and expansile. ${ }^{2}$ However, several, more aggressive cases have been reported in bone, with evidence of cortical destruction and bony expansion. Our case in particular, seemed more aggressive, and shared more similarities with a metastatic disease process, with respect to its surrounding edema and growth.

It is important to note, however, that it is difficult to characterize $\mathrm{EH}$ in the brain due to its rarity. That which has been described in bone and skin, may not apply to the intracranial compartment. In our case, each imaging tool provided us with additional diagnostic information within the spectrum of vascular-based lesions. Only after resection and subsequent pathologic analysis, were we able to reach the diagnosis of epithelioid hemangioma.

Epithelioid hemangioma is a lesion typically found in skin and bone. We present a case in which EH occurred in an extremely unusual intraparenchymal location, in the absence of clinical clues of skin or bone lesions elsewhere. The lesion mimicked a metastasis in its clinical presentation, growth, and imaging features in a patient with a history of carcinoma. Awareness of the intracranial presentation of this unusual, benign vascular entity is important as surgery is curative.

\section{Consent}

The patient described has given their informed consent for collection and potential publication of the case report.

\section{Financial disclosure}

Amin Kassam reports involvement in the Synaptive Medical Corporation (Consultant)

\section{Acknowledgements}

This research is supported by an award to the Aurora Research Institute by the Vince Lombardi Cancer Foundation. We would like to thank Nico Corporation, Carl Zeiss, Synaptive Medical, Stryker Medical, and Karl Storz for their donations that made our research possible in the Neuroanatomy Laboratory

\section{Conflict of interest}

Authors Srikant Chakravarthi, Srivathsa Veeraraghavan, Jonathan Jennings, Melanie Fukui, and Richard Rovin declare that there are no conflicts of interest regarding the publication of this paper.

\section{References}

1. Osteraas N, Cherian L, Connors J, et al. Primary Cerebral Epithelioid Hemangiomas. Neurology. 2015;84(14 Supplement):P1.039.

2. Errani C, Zhang L, Panicek DM, et al. Epithelioid hemangioma of the bone and soft tissue. A reappraisal of a controversial entity. Clin Orthop Relat Res. 2012;470(5):1498-1506.

3. Zheng J, Liu L, Wang J, et al. Primary intracranial epithelioid hemangioendothelioma: a low-proliferation tumor exhibiting clinically malignant behavior. J Neurooncol. 2012;110(1):119-127.

4. O'Connell JX, Nielson GP, Rosenberg AE. Epithelioid vascular tumors of the bone: a review and proposal of a classification scheme. Adv Anat Pathol. 2001;8(2):74-82.

5. Wenger DE, Wold LE. Malignant vascular lesions of bone: radiologic and pathologic features. Skeletal Radiol. 2000;29(11):619-631. 